\title{
Association of US Dialysis Facility Staffing with Profiling of Hospital-Wide 30-Day Unplanned Readmission
}

\author{
Yanjun Chen ${ }^{a}$ Connie Rhee $^{b}$ Damla Senturk $^{c}$ Esra Kurum $^{d}$ Luis Campos ${ }^{\mathrm{e}}$ \\ Yihao Lic Kamyar Kalantar-Zadeh ${ }^{\mathrm{b}}$ Danh Nguyen ${ }^{f}$

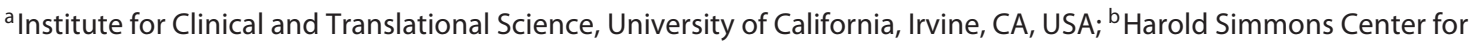 \\ Chronic Disease Research and Epidemiology, University of California Irvine School of Medicine, Orange, CA, USA; \\ 'Department of Biostatistics, University of California, Los Angeles, CA, USA; d Department of Statistics, University of \\ California, Riverside, CA, USA; ' Department of Statistics, Harvard University, Cambridge, MA, USA; ' Department of \\ Medicine, University of California Irvine, Orange, CA, USA
}

\section{Keywords}

Dialysis facility $\cdot$ End-stage renal disease $\cdot$ Profiling $\cdot$ Staffing $\cdot$ Hospital readmission

\begin{abstract}
Background: Unplanned hospital readmissions are a major source of morbidity among dialysis patients, in whom the risk of hospital readmission is exceptionally high. The contribution of dialysis facility staffing to hospital readmission has been largely overlooked. Methods: Using annual data of dialysis patients from the United States Renal Data System from 2010 to 2013, we assessed dialysis facilities with a significantly worse (SW) and facilities with a nonsignificant (NS) standardized readmission ratio (SRR). SRR estimates were risk adjusted for patient factors, past year comorbidities, and index hospitalization characteristics. Facility staffing variables were compared between 2 exposure groups: facilities with SW and NS SRRs. Four measures of staffing, including patient-to-staffing ratio, were compared between SW and matched NS facilities. Results: About 136,000-148,000 dialy-
\end{abstract}

sis patients with $269,000-319,000$ index hospital discharges were used to identify facilities with SW and facilities with NS SRR annually. Approximately 3-4\% of facilities were identified as having SW SRR among $>5,000$ facilities annually. The percent of nurses-to-total staff was significantly lower in 2010 for SW facilities than in matched NS facilities (42.5 vs. $45.6 \%, p=0.012$ ), but this disparity was attenuated by 2013 ( 44.8 vs. $44.7 \%, p=0.949$ ). There was a higher patient-tonurse ratio for SW facilities than for NS facilities (mean 16.4 vs. $15.2, p=0.038$ ) in 2010 as well, and the disparity was reduced by 2013. The trends were similar for patient-to-total staff and patient-to-registered nurse, but not statistically significant. Conclusions: This study found that dialysis facilities with SW 30-day readmission rates had lower proportions of nurses-to-total staff and higher patient-to-nurse ratios, but this disparity improved in recent years. Additional research is warranted focusing on how evidence-based staffing at dialysis facilities can contribute to reduction of hospital readmission, and this knowledge is needed to inform clinical practice guidelines and policy decisions regarding optimal dialysis patient staffing.

(c) 2019 S. Karger AG, Basel

\section{KARGER}

(c) 2019 S. Karger AG, Basel

E-Mail karger@karger.com

www.karger.com/kdd
Danh V. Nguyen, MS, PhD

Department of Medicine, University of California Irvine

333 City Blvd. West, Suite 400

Orange, CA 92868-3298 (USA)

E-Mail danhvn1@uci.edu 


\section{Introduction}

Compared to most other morbid populations, dialysis patients have a substantially higher level of mortality and morbidity, particularly with respect to hospital readmissions $[1,2]$. Dialysis patients were admitted to the hospital twice each year on average $[1,3]$. Furthermore, about $30 \%$ of hospitalized dialysis patients had an unplanned readmission within 30 days [1], with substantial implications on patient morbidity burden, health-related quality of life, and costs [4].

In recent years, the Center for Medicare and Medicaid Services (CMS) have implemented quality of care measures for 30-day readmission based on profiling models [5-10], which compare the performance of a specific health-care provider, such as a hospital or dialysis facility, to the national average rate of readmission. The performance metric used was 30-day standardized readmission ratio (SRR). Profiling models provide dialysis facilityspecific SRR estimates that are risk adjusted for patient factors, past year comorbidities, and index hospitalization characteristics $[11,12]$. In this work, we conducted the first study that examined US dialysis patients from the United States Renal Data System (USRDS) across multiple years (2010-2013) to compare dialysis facilities' staffing, including the proportion of registered nurses (RNs)to-total staff and ratios of patient-to-nurse and patientto-total staff, between facilities identified as having a significantly worse (SW) and a nonsignificant (NS) 30day SRR. Indeed, there is a current debate in the US among stakeholders, including policy makers, to mandate specific patient-to-staff ratios in dialysis facilities [13], although rigorous studies to form the evidence base are sparse.

We note that potential strategies to reduce the risk of readmission for dialysis patients are multifaceted. For example, care coordination strategies are important for postdischarge assessment and early intervention at the dialysis facility [14]. Coordination between hospital and outpatient dialysis units [15] and communication between dialysis staff, hospital staff, and physicians across both settings at the time of a patient's discharge have been discussed as target areas for improvement [16-18]. Our work here focuses on another modifiable factor, namely the patient care staffing at dialysis facilities. Indeed, staffing ratios and composition vary substantially across dialysis facilities [19]. We use a rigorous 2-stage analysis to identify SW and NS facilities in stage 1 and an independent comparative analysis of facility staffing characteristics between SW and NS facilities in stage 2 .

\section{Methods}

Study Cohort and Data Source

The source data for the study was from the USRDS, a national registry that includes nearly all patients receiving care for endstage renal disease (ESRD) in the US [20] from the years 20102013 (January 1, 2010, to December 31, 2013). Annual study cohort sizes are provided in online supplementary Figure 1 (see www.karger.com/doi/10.1159/000496147 for all online suppl. material). Patient-level data were collected from patient profile, payer history, Medical Evidence Form (CMS-2728), transplant, patient treatment history files, institutional claims files, and hospitalization standard analytic files. Facility-level data, including staffing and the number of patients receiving care, were obtained from the ESRD facility survey (CMS-2744). The study was approved by the University of California Irvine Institutional Review Board.

\section{Index Discharges and Inclusion/Exclusion Criteria}

Hospital data through December 31, 2013, were available for analysis. Hospitalizations with admission dates from January 1 through December 31 of each year were used. Index discharges were restricted to Medicare-covered hospitalization events at acute care hospitals. Index discharges were restricted to patients who were covered by Medicare as primary payer at day 91 from start of dialysis, because USRDS hospitalization data are incomplete for non-Medicare patients and for patients classified as Medicare as secondary payer [20]. Each hospital index discharge date was linked to the dialysis facility under which the patient was receiving dialysis care.

Exclusion criteria for index discharge included the following. Hospitalizations with length of stay longer than 365 days were excluded (unusual cases), and duplicate hospitalizations (identified as the same patient admitted to the same hospital with the same admission and discharge date) were removed. If a patient had more than 12 inpatient stays within 1 year, hospitalizations that occurred after the patient's 12th hospital admission within each year were excluded [11]. This cap in the readmission has been advocated to address concerns that allowing $>12$ discharges per person would unfairly affect smaller facilities [11]; however, because there were $<1 \%$ of these cases, the impact was small [11]. Since index discharges are hospitalizations at acute care hospitals, discharges from skilled nursing facilities, long-term care hospitals, rehabilitation hospitals, and hospice were excluded. Hospitalizations for patients who were discharged against medical advice or transferred to another acute care facility were excluded as these do not capture unplanned readmission. In-hospital deaths were excluded as well because there would be no readmission. In addition, hospitalizations where patients were admitted for cancer, primary psychiatric diagnosis, and rehabilitation were excluded [11] as these events are not eligible as unplanned readmission. Also, a hospitalization was excluded if the patient died within 30 days from discharge because there was no opportunity for 30-day readmission.

An extremely small facility with several patients is not appropriate for profiling analysis to assess the facility's readmission rate; therefore, facilities with $<10$ index discharges were excluded. Also, when a facility's raw readmission rate (calculated as the percentage of 30-day readmission among all index discharges) equaled 1 (i.e., all index hospitalizations resulted in readmissions within 30 days), the facility was excluded. This occurred for extremely small facili- 
ties with 1-4 patients. Dialysis facility exclusion is documented in online supplementary Table 1.

\section{Outcomes}

The outcome was all-cause unplanned readmission to an acute care hospital within 30 days after the date of an index hospitalization discharge in stage 1 . A readmission was designated as planned or unplanned following CMS methodology for all-cause 30-day readmission $[8,11]$. Planned readmissions were defined as readmissions that were either nonacute or for a maintenance care/therapy. More specifically, a prespecified list of 32 planned procedures was defined using the full Agency for Healthcare Research and Quality Clinical Classification System procedure category (see online suppl. Table 2A) [8]. Admissions for an acute illness or for complications of care were not "planned." Discharge condition categories considered as acute or complications of care were listed in supplementary Table 2B [8]. Only admissions during which at least 1 of the 32 listed procedures were performed, and concerning patients who were not admitted for an acute illness or complications of care, were considered "planned," otherwise they were considered unplanned.

Four patient care staffing outcomes were considered in stage 2 analysis, which compared dialysis facilities' staffing between facilities with SW and NS 30-day readmissions: (1) percent of nurses-to-total staff, (2) patient-to-nurse ratio, (3) patient-to-RN ratio, and (4) patient-to-total staff ratio. The number of RNs included the number of full-time RNs plus part-time RNs, but a part-time $\mathrm{RN}$ was counted as one-half RN. The number of nurses was defined to be the number of RNs and licensed practical nurses, and total staff included RNs, licensed practical nurses, and patient care technicians. The number of patients was defined as the number of dialysis outpatients at the end of the year.

\section{Covariates and Exposure}

Profiling analysis in stage 1 aims to estimate the facility-specific SRR, adjusted for patient risk factors/covariates. The rationale for the categories of risk adjustment for 30-day hospital-wide readmission and their development has been reported $[8,9,11]$. Similarly, in profiling models here we adjusted for age at hospitalization, sex, body mass index, diabetes as the cause of ESRD, years on dialysis, length of index hospitalization, high-risk index hospitalization, and past-year comorbidities (online suppl. Table 3). Pastyear comorbidities were created by defining unique ICD-9 diagnosis codes from institutional claims within 1 year before admission dates. Five claim sources were used: inpatient, outpatient, skilled nursing facility, hospice, and home health claims. These diagnosis codes were grouped by using the Department of Health and Human Services' hierarchical condition categories [21]. Pastyear comorbidities are listed in online supplementary Table 3. Finally, high-risk diagnosis was defined as any diagnosis area that was extremely rare but had a 30-day readmission rate of at least $40 \%$ using 11 Clinical Classification System areas identified as high risk [21] (online suppl. Table 2C). In stage 2 analyses (facility level), the exposure groups were SW and NS facilities.

\section{Statistical Analysis}

Stage 1

Profiling models that provide effective risk adjustment include random effects (RE) $[8,10]$ and fixed effects (FE) $[11,12,22]$ hierarchical logistic regression models (HLRMs). In stage 1 analysis,
HLRMs were used to profile dialysis facilities using 30-day readmissions as the outcome, adjusted for patient risk factors. The HLRM was used to estimate the risk-adjusted SRR for each dialysis facility. SRR is the ratio of the number of readmissions for a specific facility to the expected number of readmissions assuming a national average and adjusted for patient-level risk factors. We considered both RE and FE models. For FE models, we examined 2 FE models, FE1 and FE2, where FE2 adds a RE to the FE1 model to account for additional variation in discharging hospitals [12]. Each profiling model was used to flag SW and NS facilities. For the RE model, a bootstrap based on 500 resampling of facilities with replacement was used to obtain $95 \%$ confidence intervals (CIs) for SRRs $[8,10]$. For FE models, inference was based on testing the null hypothesis that SRR $=1$ through 500 resampling under the null with empirical null adjustment [22, 23].

Stage 2

To allow for risk adjustment required in stage 1 and to avoid confounding of the patient care process variables with patient risk factors and outcome (readmission), facilities that have SW and NS readmissions were independently identified by profiling models in stage 1 . However, a direct comparison of patient care staffing variables between SW and NS facilities is not appropriate because of differences in patient case-mix characteristics, including comorbidities. Therefore, in stage 2, we first matched facilities with NS readmissions to facilities with SW readmissions with respect to average patient risk factors as well as facility size (number of patients). Matching was achieved using the propensity score [24, 25], and adequacy of matching was assessed by checking the balances of covariates before and after matching using the absolute standardized difference criteria [26]. Comparisons of patient staffing variables between matched sets of SW and NS facilities were based on multiple linear regression models (to doubly adjust for average patient risk factors). Finally, a secondary analysis to assess changes in patient staffing over time was based on linear mixed effects models. SAS/ STAT $^{\circledR}$ version 9.4 was used for all analyses [27], except for RE and FE profiling models which were implemented in R version 3.4 [28].

\section{Results}

\section{Study Cohorts}

The study cohort for each year comprised about 146,000-148,000 patients with raw unplanned 30-day readmission rates of about $30 \%$ among approximately 310,000-319,000 index discharges (online suppl. Table 4 ). For each year, over 5,000 dialysis facilities were included for profiling analysis.

Characteristics of patient risk factors prior to index hospitalization are summarized in online supplementary Table 3. For example, the 2013 cohort comprised $63 \%$ of patients aged 45-75 years and 48\% females. Approximately $24,15,12,25$, and $23 \%$ of patients were on ESRD for $<1,1-2,2-3,3-6$, and $>6$ years, respectively. Diabetes was the cause of ESRD in about $43 \%$ of patients. High-risk index hospitalizations accounted for about 1\% of hospi- 


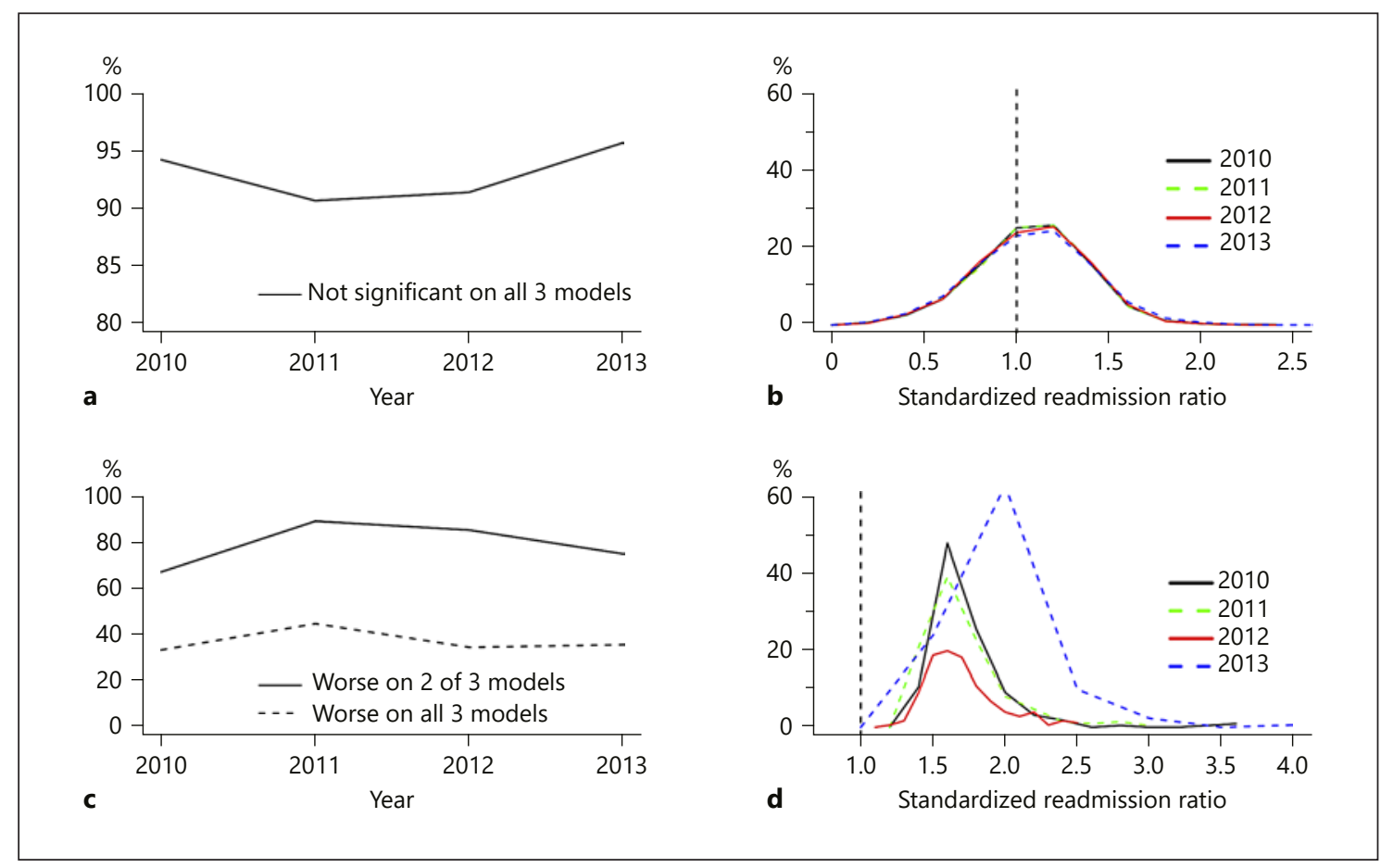

Fig. 1. Dialysis facilities flagged by profiling models in 2010-2013. a Dialysis facilities flagged as nonsignificant (denominator is the total number of facilities in each year from 2010 to 2013 : $N_{F}=5,346,5,579,5,637$, and 5,628). b Among facilities flagged as significantly worse (SW) by at least 1 model $\left(2010-2013: N_{F}=222,211,176\right.$, and 171); the percentage flagged as SW by all 3 models is presented. Distribution of estimated standardized readmission ratio for facilities flagged as nonsignificant $(\mathbf{c})$ and SW (d).

talizations, and 47,22 , and $31 \%$ of hospitalizations were for $<5,5$ or 6 , and $>6$ days in duration, respectively. Pastyear comorbidities prior to index hospitalization are also summarized in online supplementary Table 3 . The most common past-year comorbidities were chronic obstructive pulmonary disease (26\%), cardiorespiratory failure/ shock $(22 \%)$, coagulation defects and other specified hematological disorders (16\%), other infectious disease and pneumonias (41\%), septicemia/shock (17\%), and psychiatric comorbidity (29\%).

Stage 1: Identifying Dialysis Facilities with SW SRR

Profiling models identified facilities with SW and NS risk-adjusted SRRs. Profiling results for the 2010 cohort are summarized in online supplementary Table 5. Approximately $94-96 \%$ of all facilities were flagged as NS by all 3 models across years; i.e., $94-96 \%$ of facilities had SRRs not different from the national average. Highlighted in online supplementary Table 5 are the flagging results for the 2010 cohort with $94 \%\left(N_{F}=5,043\right)$ NS facilities and $N_{F}=222$ facilities flagged as having SW SRRs by at least 1 of the 3 models. Figure $1 \mathrm{a}, \mathrm{b}$ shows the results over time with respect to facilities flagged as NS (Fig. 1a) and SW (Fig. 1b). We note that the overall rate of facilities flagged as SW by at least 1 model was similar for 2010-2013: 4.2\% (222/5,346), 3.8\% (211/5,579), 3.1\% $(176 / 5,637)$, and $3.0 \%(171 / 5,628)$, respectively. The estimated SRRs for facilities that were NS and SW are displayed in Figure 1c and d, respectively. For SW facilities, the 5th and 95th percentiles of the estimated SRRs were 1.3 and 2.1 in 2010, respectively, and the distributions of SW SRRs were similar across years.

Trends in the adjusted odds ratio (OR) and associated 95\% CIs for patient risk factors are summarized in online supplementary Figure 2 for the 2011 cohort. Most pastyear comorbidities were associated with significantly higher odds of 30-day readmission, except for 4 comorbid groups: hip fracture, transplant, respiratory dependence, and rheumatoid arthritis/inflammatory connective tissue disease. Significant risk factors associated with 9\% (OR 1.09 , 95\% CI 1.05-1.13), 27\% (OR 1.27, 95\% CI 1.26-1.29), and $40 \%$ (OR 1.40, 95\% CI 1.35-1.45) higher odds of readmission were motor impairment, other infectious disease, and drug or alcohol disorders (online suppl. Fig. 2A). 


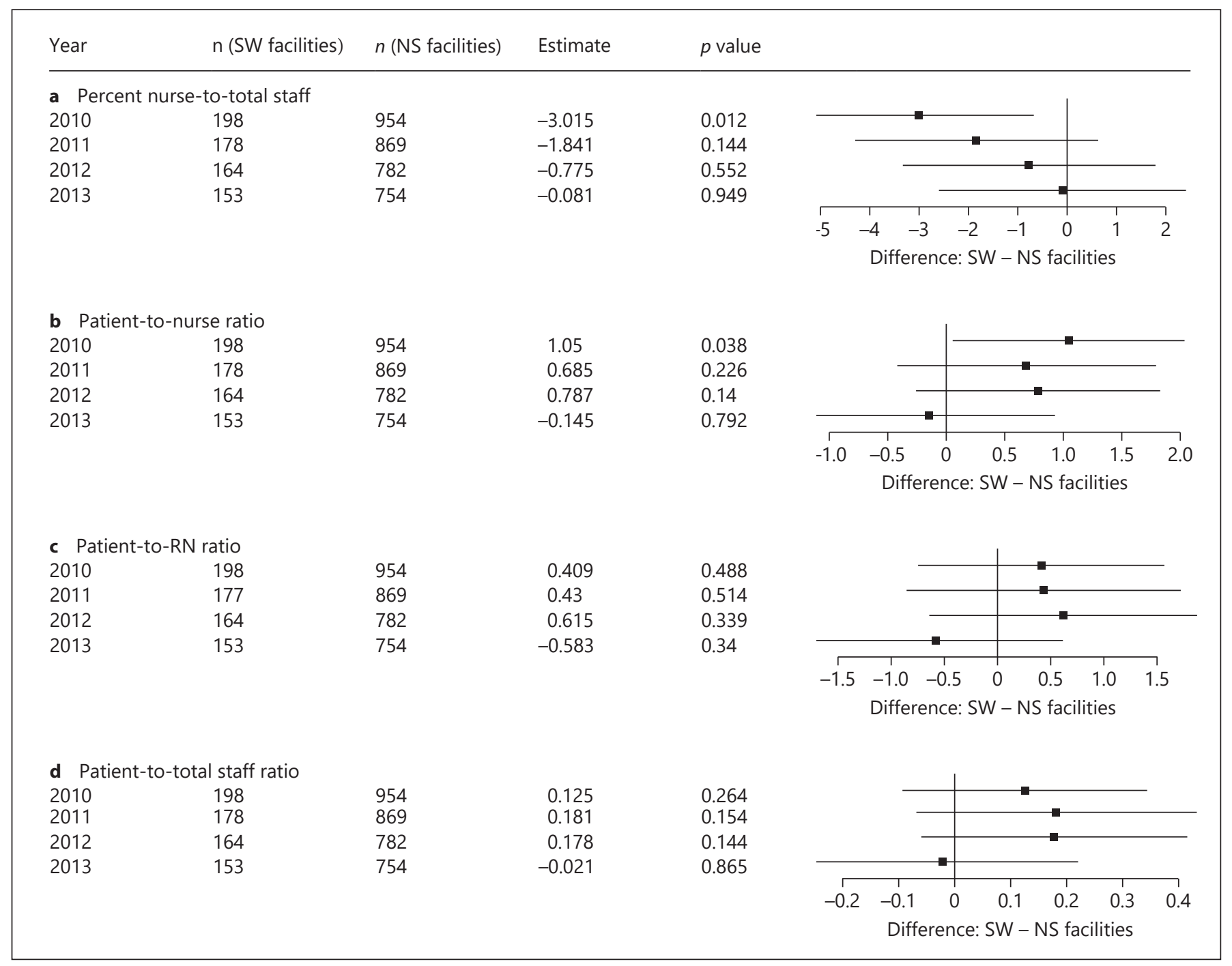

Fig. 2. Comparison of patient staffing characteristics in dialysis facilities with significantly worse (SW) readmission versus facilities with nonsignificant (NS) readmission in the years 2010-2013 ( $n$ = number of facilities). RN, registered nurses.

Females, younger age groups ( $<25$ and $25-45$ years) relative to the $45-60$ years reference group, diabetes as the cause of ESRD, high-risk index discharge, and longer index hospitalization were associated with higher risk of readmission (online suppl. Fig. 2B). These trends in the adjusted ORs were similar for the 2012 through 2013 cohorts (results not shown).

\section{Stage 2: Comparison of Dialysis Facility Patient Care Staffing}

In stage 2 analysis, we compared dialysis facilities' staffing levels and patient-to-staff composition between SW and NS facilities. The numbers of facilities with SW
SRRs identified from stage 1 were 222, 211, 176, and 171 for the years 2010-2013, respectively. We removed extreme outlier facilities. Specifically, facilities with nurses, total staff, and patient-to-total staff ratios greater than the 99.5th percentile, or with patient-to-total staff ratios that were smaller than the 0.5 th percentile, which appeared to include database errors, were excluded. Also, similar to previous work [19], facilities with 0 nurses were not included. This resulted in $N_{\mathrm{F}}=198,178,164$, and $153 \mathrm{SW}$ facilities (years 2010-2013, respectively) used in stage 2 analysis. As described above, a large number of facilities with NS SRR were identified ( $>5,000$ facilities); however, patient case-mix characteristics, including comorbidities, 
Table 1. Summary statistics of staffing variables for a matched set of significantly worse facilities and nonsignificant facilities for the 2010-2013 cohorts

\begin{tabular}{|c|c|c|c|c|c|c|c|}
\hline \multirow[t]{2}{*}{ Year } & \multirow[t]{2}{*}{ Variable } & \multicolumn{3}{|c|}{ Significantly worse facilities } & \multicolumn{3}{|c|}{ Nonsignificant facilities } \\
\hline & & $n$ & mean & SD & $n$ & mean & $\mathrm{SD}$ \\
\hline \multirow[t]{4}{*}{2010} & patient-to- $\mathrm{RN}$ ratio & 198 & 18.61 & 7.18 & 954 & 18.11 & 7.85 \\
\hline & patient-to-nurse ratio & 198 & 16.36 & 6.38 & 954 & 15.24 & 6.63 \\
\hline & patient-to-total staff ratio & 198 & 6.28 & 1.45 & 954 & 6.15 & 1.45 \\
\hline & percent of nurses-to-total staff & 198 & 42.50 & 14.56 & 954 & 45.60 & 15.92 \\
\hline \multirow[t]{4}{*}{2011} & patient-to- $\mathrm{RN}$ ratio & 177 & 18.51 & 7.79 & 869 & 17.97 & 8.36 \\
\hline & patient-to-nurse ratio & 178 & 16.30 & 6.99 & 869 & 15.51 & 7.18 \\
\hline & patient-to-total staff ratio & 178 & 6.34 & 1.69 & 869 & 6.15 & 1.59 \\
\hline & percent of nurses-to-total staff & 178 & 43.38 & 15.22 & 869 & 45.45 & 16.49 \\
\hline \multirow[t]{4}{*}{2012} & patient-to-RN ratio & 164 & 17.50 & 7.14 & 782 & 16.82 & 7.82 \\
\hline & patient-to-nurse ratio & 164 & 15.25 & 6.61 & 782 & 14.44 & 6.34 \\
\hline & patient-to-total staff ratio & 164 & 6.09 & 1.31 & 782 & 5.93 & 1.52 \\
\hline & percent of nurses-to-total staff & 164 & 45.43 & 16.01 & 782 & 46.35 & 15.98 \\
\hline \multirow[t]{4}{*}{2013} & patient-to- $\mathrm{RN}$ ratio & 153 & 16.66 & 6.56 & 754 & 17.29 & 7.26 \\
\hline & patient-to-nurse ratio & 153 & 14.87 & 6.08 & 754 & 15.04 & 6.40 \\
\hline & patient-to-total staff ratio & 153 & 5.96 & 1.33 & 754 & 5.98 & 1.44 \\
\hline & percent of nurses-to-total staff & 153 & 44.83 & 15.41 & 754 & 44.75 & 15.07 \\
\hline
\end{tabular}

SD, standard deviation; RN, registered nurses.

in these NS facilities differed from SW facilities (online suppl. Fig. 3 - before matching). Thus, we first matched NS facilities to SW facilities on average patient age, facility size, percent of high-risk discharges, frequencies of past-year comorbidities, and all other risk factors presented in online supplementary Table 3. A maximum of 5 NS facilities was matched with each SW facility. In 93\% of cases, the SW:NS match was 1 SW:5 NS facilities, while for the remaining 7\%, the match was 1 SW:1-4 NS facilities. Online supplementary Figure 3 shows the adequacy of matching results with absolute standardized differences $<0.1$.

Patient staffing level and ratios between matched SW and NS facilities are summarized in Table 1. In 2010, the average (SD) percent of nurses-to-total staff was $42.5 \%$ (14.6\%) for SW facilities and 45.6\% (15.9\%) for NS facilities $(p=0.012$; Fig. $2 \mathrm{a})$, and the model-based estimate of the average percentage of nurses-to-total staff was 3.0\% lower for SW facilities (Fig. 2a). The observed disparity in the average percentage of nurses-to-total staff between SW and NS facilities was reduced in the year 2011 and was nearly equalized by 2013 : mean $44.8 \%$ (15.4\%) for SW facilities and $44.8 \%(15.1 \%)$ for NS facilities ( $p=0.949$; Table 1; Fig. 2a).
The average (SD) patient-to-nurse ratio was higher by 1.1 for SW than for NS facilities: mean 16.4 (6.4) versus 15.2 (6.6) ( $p=0.038$; Table 1; Fig. 2b). The average (SD) patient-to-nurse ratios in SW and NS facilities were similar in 2011 and 2012, although in 2013 the difference between SW and NS facilities was negligible: mean 14.9 (6.1) for SW facilities versus 15.0 (6.4) for NS facilities ( $p=$ 0.792; Table 1; Fig. 2b). For patient volume relative to RNs, the overall pattern of results was similar between SW and NS facilities, although not statistically significant. For example, the average (SD) patient-to-RN ratio was 17.5 (7.1) for SW facilities versus 16.8 (7.8) for NS facilities by $2012(p=0.339$; Fig. $2 c)$ and was also similar in 2013.

A trend of SW facilities having a higher average patient-to-total staff ratio, based on the pattern of $95 \%$ CIs (Fig. 2d), was also observed in 2010-2012. In 2013, the difference in patient-to-staff ratio between SW and NS facilities was negligible.

Finally, in a secondary analysis we assessed the facility staffing characteristics' longitudinal trend using linear mixed effects models. We found that relative to 2010, there has been an overall significant decline in patient-to$\mathrm{RN}$, patient-to-nurse, and patient-to-staff ratios, while 
Table 2. Linear mixed effects model for facility staffing characteristics assessing longitudinal trend within matched sample

\begin{tabular}{|c|c|c|c|c|}
\hline Outcome & Comparison & Estimate & Standard error & $p$ value \\
\hline Patient-to- $\mathrm{RN}$ ratio & $\begin{array}{l}2011 \text { versus } 2010 \\
2012 \text { versus } 2010 \\
2013 \text { versus } 2010\end{array}$ & $\begin{array}{l}-0.33 \\
-1.19 \\
-1.24\end{array}$ & $\begin{array}{l}0.11 \\
0.11 \\
0.11\end{array}$ & $\begin{array}{r}0.003 \\
<0.001 \\
<0.001\end{array}$ \\
\hline Patient-to-nurse ratio & $\begin{array}{l}2011 \text { versus } 2010 \\
2012 \text { versus } 2010 \\
2013 \text { versus } 2010\end{array}$ & $\begin{array}{l}-0.08 \\
-0.66 \\
-0.68\end{array}$ & $\begin{array}{l}0.08 \\
0.08 \\
0.08\end{array}$ & $\begin{array}{r}0.352 \\
<0.001 \\
<0.001\end{array}$ \\
\hline Patient-to-total staff ratio & $\begin{array}{l}2011 \text { versus } 2010 \\
2012 \text { versus } 2010 \\
2013 \text { versus } 2010\end{array}$ & $\begin{array}{l}-0.05 \\
-0.21 \\
-0.21\end{array}$ & $\begin{array}{l}0.02 \\
0.02 \\
0.02\end{array}$ & $\begin{array}{r}0.037 \\
<0.001 \\
<0.001\end{array}$ \\
\hline Percent of nurses-to-total staff & $\begin{array}{l}2011 \text { versus } 2010 \\
2012 \text { versus } 2010 \\
2013 \text { versus } 2010\end{array}$ & $\begin{array}{l}0.01 \\
0.26 \\
0.32\end{array}$ & $\begin{array}{l}0.16 \\
0.16 \\
0.16\end{array}$ & $\begin{array}{l}0.947 \\
0.099 \\
0.041\end{array}$ \\
\hline
\end{tabular}

$\mathrm{RN}$, registered nurses.

there was an increase in the percent of nurses relative to total patient care staff (Table 2).

We note that the main interest in profiling analysis centers on identifying SW facilities compared to NS facilities. However, although typically not considered, we also examined dialysis facilities identified with significantly better (SB) hospital readmission rates (i.e., readmission rates significantly lower than the national average). The results show that staffing characteristics for SB facilities were not different from NS facilities (online suppl. Fig. 4). Thus, the pattern of results described above was observed in SW facilities only and not in SB facilities.

\section{Discussion}

Unplanned hospital readmissions are a major health burden to patients and cost to the health-care system, and even more so for dialysis patients given the substantially higher rate of 30-day unplanned readmission compared to the general population [3]. Using the national data and rigorous patient-level profiling models, we identified dialysis facilities that had SW and NS annual readmission rates. After matching to eliminate differences in patient characteristics (and facility size) between SW and NS facilities, we found that SW facilities had higher ratios of patient-to-nurse and lower nurses-to-total staff, particularly in earlier years (e.g., 2010). These disparities appeared to have been attenuated by 2013 . As national pa- tient-level data are released in the coming years, it would be of interest to monitor these encouraging trends in patient staffing ratios.

Also, although qualitative, we note that the average ratios of patient-to-RN, patient-to-nurse, and patient-tototal staff all declined from 2010 to 2013 (Table 1, 2), similar to the decline in the overall rate of SW facilities (from $4.2,3.8$, and 3.1 to $3.0 \%$ from 2010 to 2013 , respectively). Furthermore, during these years, there was an increase in the percent of nurses-to-total staff (from 42.5 to $44.8 \%$ ) as well.

Preventing recurrent hospitalizations in dialysis patients is a complex process that involves multiple areas, including (1) managing patients' medical issues before, during, and after hospitalization (e.g., anemia, malnutrition, volume overload, mineral metabolism disorders, and infection) [17]; and (2) timely communication and coordination of care between hospital and ambulatory dialysis facility staff and physicians. Patients may be prescribed new therapies or undergo changes in their health parameters over the course of their hospitalization that require monitoring and/or adjustment to their prior management approaches upon returning to their outpatient dialysis facility. Furthermore, given their frequency of interaction, ambulatory dialysis staff and physicians may be the first providers to assess patients following hospital discharge, including medication reconciliation [18, $29,30]$. Also, critical health parameters, including patients' anemia (e.g., hemoglobin), serum albumin, and 
mineral bone disease (e.g., calcium, phosphorus, and parathyroid hormone) as well as dry weights may have substantially changed over the course of hospitalization, and in prior studies of dialysis patients these parameters were found to be significantly decreased after hospitalization [14]. Therefore, care coordination and discharge planning are paramount for timely implementation of posthospitalization care, optimization of health status, and early identification and intervention for recurrent illness immediately following discharge. Improved patient care coordination and intervention to reduce readmission has also been examined extensively in the non-ESRD populations [31-37]. Thus, adequate dialysis facility staffing is critical.

Our work here systematically examined a largely overlooked factor, specifically the dialysis patient staffing ratio and infrastructure within dialysis facilities, as a contributor to unplanned hospital readmissions. Staffing levels and composition in dialysis facilities are ultimately modifiable factors that can be optimized and thereby improve patient outcomes [38, 39]. Structural staffing issues, specifically inadequate nurse staffing in acute care hospitals, have been linked to heightened risk of infection [40], mortality [41], and other adverse consequences [4245]. Adequacy of dialysis clinic staffing and quality of care are linked [46], although what constitutes "adequate" staffing at dialysis facilities has not yet been defined, particularly with respect to ameliorating hospitalization risk [47]. Indeed, there is urgent need for further study of the optimal dialysis patient-to-staffing ratios given recent controversy with respect to legislation regarding mandated dialysis staffing ratios in ambulatory facilities. For example, in the state of California serving 63,000 dialysis patients across 560 freestanding dialysis clinics, there has been a proposal of a State Senate Bill, the "Dialysis Patient Safety Act" (SB 349 [48, 49]), which would mandate dialysis patient-to-nurse ratios of $8: 1$, as well as patient-totechnician and patient-to-social worker ratios of $3: 1$ and $75: 1$, respectively. While this would be the first US law to mandate dialysis staffing ratios, other states have set minimum staffing requirements via administrative regulations. Hence, rigorous studies are needed to determine adequate dialysis facility staffing ratios associated with optimal patient outcomes in order to guide stakeholders, including policy makers, regulatory bodies, patients, and dialysis facility providers [13].

Our study utilized a 2-stage analysis to avoid confounding of the patient care process (staffing) variables with patient risk factors and the outcome, 30-day readmissions. In the first stage, a profiling model (hierarchical logistic regression) was used to estimate facility effects (contribution of a facility total process to hospital readmission of patients at that facility). We note that a common misunderstanding in profiling analysis centers on the intuition to include (adjust for) factors related to the process of care, such as the effect of nephrologist or nephrology care or other facility-level factors, including management strategies (e.g., infection control policies). No facility-level factors or no factors on the "causal pathway" after the start of follow-up for a patient (e.g., level of nephrologist care) should be included as a basis to assess SRR [10-12, 50] because doing so would explain away variation in outcomes presumably attributable to dialysis facilities. However, there are several limitations. A limitation of our study is the quality of the self-reported data from the annual facility survey due to potential reporting errors or data entry errors. Additionally, we did not examine potential contributions of dietitians and social workers employed at dialysis facilities because $>75 \%$ of dialysis facilities did not report or reported $\leq 1$ dietitian or social worker. Also, lack of data precluded an examination of factors such as patient-to-nephrologist ratio and dialysis nurses' level of experience in stage 2 analysis.

Our study focused on SRR and associated staffing ratios in the US dialysis population. Similar analyses in other populations, such as other countries/regions, would facilitate interesting comparative studies. Towards this objective, establishment of appropriate population registries that include hospitalization is needed.

In summary, this study found that dialysis facilities with SW 30-day hospital readmissions have lower proportions of nurses-to-total staff and higher patient-tonurse ratios. However, the disparities in staffing characteristics between SW and NS facilities were attenuated by 2013. Although there were trends of higher patient-toRN and higher patient-to-total staff ratios in facilities with SW SRRs, these were not statistically significant. Annual monitoring/profiling analysis, as described here, is a useful tool to see whether this trend continues or reverses over time as data become available. Finally, the evidence from this study does not definitely support or not support mandated staffing ratios. Rather, it highlights the critical need to develop the evidence base of how patient care staffing at dialysis facilities can contribute to a reduction of hospital readmissions, and this knowledge is needed to inform clinical practice guidelines and policy decisions regarding optimal dialysis patient staffing, which currently are lacking. 


\section{Statement of Ethics}

The study was approved by the University of California Irvine Institutional Review Board.

\section{Disclosure Statement}

K.K.-Z. has served as a medical director of FMC and Davita dialysis clinics. The other authors declare that they have no other relevant financial interests.

\section{Funding Sources}

This study was supported by NIDDK grants R01 DK092232 (Drs. Senturk, Nguyen, Kalantar-Zadeh, and Rhee) and K23 DK102903 (Drs. Rhee, Kalantar-Zadeh, and Nguyen). The interpretation/reporting of the data presented are the responsibility of the authors and in no way should be seen as an official policy or interpretation of the US government. The study sponsors did not have a role in the study design; collection, analysis, or interpretation of data; writing the report; or the decision to submit the report for publication.

\section{References}

1 United States Renal Data System. 2016 USRDS annual data report: Epidemiology of kidney disease in the United States. Bethesda (MD): National Institutes of Health, National Institute of Diabetes and Digestive and Kidney Diseases; 2016

2 de Jager DJ, Grootendorst DC, Jager KJ, van Dijk PC, Tomas LM, Ansell D, et al. Cardiovascular and noncardiovascular mortality among patients starting dialysis. JAMA. 2009 Oct;302(16):1782-9.

3 U.S. Renal Data System. USRDS 2012 Annual Data Report: Atlas of Chronic Kidney Disease and End-Stage Renal Disease in the United States. Bethesda (MD): National Institutes of Health, National Institute of Diabetes and Digestive and Kidney Diseases; 2012.

4 U.S. Renal Data System. USRDS 2013 Annual Data Report: Atlas of Chronic Kidney Disease and End-Stage Renal Disease in the United States. Bethesda (MD): National Institutes of Health, National Institute of Diabetes and Digestive and Kidney Diseases; 2013.

5 Keenan PS, Normand SL, Lin Z, Drye EE, Bhat KR, Ross JS, et al. An administrative claims measure suitable for profiling hospital performance on the basis of 30-day all-cause readmission rates among patients with heart failure. Circ Cardiovasc Qual Outcomes. 2008 Sep;1(1):29-37.

6 Krumholz HM, Lin Z, Drye EE, Desai MM, Han LF, Rapp MT, et al. An administrative claims measure suitable for profiling hospital performance based on 30-day all-cause readmission rates among patients with acute myocardial infarction. Circ Cardiovasc Qual Outcomes. 2011 Mar;4(2):243-52.

7 Lindenauer PK, Normand SL, Drye EE, Lin Z, Goodrich K, Desai MM, et al. Development, validation, and results of a measure of 30-day readmission following hospitalization for pneumonia. J Hosp Med. 2011 Mar;6(3):142-50.

8 Horwitz L, Partovain C, Lin ZQ, Herrin J, Grady J, Conover M, et al. Hospital-wide (allcondition) 30 day risk-standardized readmission measure. 2011 [cited 2016 Jun 16]. Available from: https://www.cms.gov/Medicare/ Quality-Initiatives-Patient-Assessment-Instruments/MMS/downloads/MMSHospitalWideAll-ConditionReadmissionRate.pdf
9 Horwitz LI, Partovian C, Lin Z, Grady JN, Herrin J, Conover M, et al. Development and use of an administrative claims measure for profiling hospital-wide performance on 30 day unplanned readmission. Ann Intern Med. 2014 Nov; 161(10 Suppl):S66-75.

10 Ash AS, Fienberg SE, Louis TA, Normand ST, Stukel TA, Utts J. Statistical issues in assessing hospital performance (2012 COPSS-CMS White Paper). 2012 [cited 2018 Feb 22]. Available from: https://www.cms.gov/Medicare/ Quality-Initiatives-Patient-Assessment-Instruments/HospitalQualityInits/Downloads/ Statistical-Issues-in-Assessing-Hospital-Performance.pdf

11 Centers for Medicare and Medicaid Services (CMS)/UM-KECC. Report for the standardized readmission ratio. 2014 [updated 2017] [cited 2018 Feb 20]. Available from: https:// www.cms.gov/Medicare/Quality-InitiativesPatient-Assessment-Instruments/ESRDQIP/ Downloads/MeasureMethodologyReportfortheProposedSRRMeasure.pdf

12 He K, Kalbfleisch JD, Li Y, Li Y. Evaluating hospital readmission rates in dialysis facilities; adjusting for hospital effects. Lifetime Data Anal. 2013 Oct;19(4):490-512.

13 Harrison L. California first to address dialysis staffing problems. Medscape. 2017;Epub April 21 [cited 2018 Feb 20]. Available from: https://www.medscape.com/viewarticle/878870

14 Chan KE, Lazarus JM, Wingard RL, Hakim RM. Association between repeat hospitalization and early intervention in dialysis patients following hospital discharge. Kidney Int. 2009 Aug;76(3):331-41.

15 Reilly JB, Marcotte LM, Berns JS, Shea JA. Handoff communication between hospital and outpatient dialysis units at patient discharge: a qualitative study. Jt Comm J Qual Patient Saf. 2013 Feb;39(2):70-6.

16 Castner D. Management of patients on hemodialysis before, during, and after hospitalization: challenges and suggestions for improvements. Nephrol Nurs J. 2011 Jul-Aug;38(4): 319-30.

17 Plantinga LC, Jaar BG. Preventing repeat hospitalizations in dialysis patients: a call for action. Kidney Int. 2009 Aug;76(3):249-51.
18 Wish JB. The role of 30-day readmission as a measure of quality. Clin J Am Soc Nephrol. 2014 Mar;9(3):440-2.

19 Yoder LA, Xin W, Norris KC, Yan G. Patient care staffing levels and facility characteristics in U.S. hemodialysis facilities. Am J Kidney Dis. 2013 Dec;62(6):1130-40.

20 United States Renal Data System. 2014 Researcher's Guide to the USRDS Database. ADR Edition. Bethesda (MD): National Institutes of Health, National Institute of Diabetes and Digestive and Kidney Disease, 2014 [cited 2018 Mar 11]. Available from: https://www. usrds.org/2014/rg/USRDS_Researchers_ Guide-14.pdf

21 Pope GC, Kautter J, Ellis RP, Ash AS, Ayanian JZ, Lezzoni LI, et al. Risk adjustment of Medicare capitation payments using the CMSHCC model. Health Care Financ Rev. 2004; 25(4):119-41.

22 Kalbfleisch JD, Wolfe RA. On monitoring outcomes of medical providers. Stat Biosci. 2013;5(2):286-302.

23 Efron B. Large-scale simultaneous hypothesis testing: the choice of a null hypothesis. J Am Stat Assoc. 2004;99(465):96-104.

24 Rosenbaum PR, Rubin DB. The central role of the propensity score in observational studies for causal effects. Biometrika. 1983;70(1):4155.

25 Rubin DB, Thomas N. Combining propensity score matching with additional adjustments for prognostic covariates. J Am Stat Assoc. 2000;95(450):573-85.

26 D’Agostino RB Jr. Propensity score methods for bias reduction in the comparison of a treatment to a non-randomized control group. Stat Med. 1998 Oct;17(19):2265-81.

27 SAS/STAT ${ }^{\circledR}$ version 9.4. SAS Institute Inc. Cary, NC, USA.

28 R Core Team. R: A language and environment for statistical computing. Vienna, Austria: R Foundation for Statistical Computing; 2017. Available from: https://www.R-project.org/

29 Coleman EA, Smith JD, Raha D, Min SJ. Posthospital medication discrepancies: prevalence and contributing factors. Arch Intern Med. 2005 Sep;165(16):1842-7.
Staffing in US Dialysis Facilities and 30-Day Hospital Readmission
Kidney Dis 2019;5:153-162 DOI: $10.1159 / 000496147$ 
30 Wingard RL, Chan KE, Hakim R. RightReturn. Partnering to reduce the high rate of hospital readmission for dialysis-dependent patients. Nephrol News Issues. 2012 Mar; 26(3):20-2.

31 Balaban RB, Weissman JS, Samuel PA, Woolhandler S. Redefining and redesigning hospital discharge to enhance patient care: a randomized controlled study. J Gen Intern Med. 2008 Aug;23(8):1228-33.

32 Coleman EA, Smith JD, Frank JC, Min SJ, Parry C, Kramer AM. Preparing patients and caregivers to participate in care delivered across settings: the Care Transitions Intervention. J Am Geriatr Soc. 2004 Nov;52(11): 1817-25.

33 Coleman EA, Parry C, Chalmers S, Min SJ. The care transitions intervention: results of a randomized controlled trial. Arch Intern Med. 2006 Sep;166(17):1822-8.

34 Koehler BE, Richter KM, Youngblood L, Cohen BA, Prengler ID, Cheng D, et al. Reduction of 30-day postdischarge hospital readmission or emergency department (ED) visit rates in high-risk elderly medical patients through delivery of a targeted care bundle. J Hosp Med. 2009 Apr;4(4):211-8.

35 Naylor M, Brooten D, Jones R, LavizzoMourey R, Mezey M, Pauly M. Comprehensive discharge planning for the hospitalized elderly. A randomized clinical trial. Ann Intern Med. 1994 Jun;120(12):999-1006.

36 Parry C, Min SJ, Chugh A, Chalmers S, Coleman EA. Further application of the care transitions intervention: results of a randomized controlled trial conducted in a fee-for-service setting. Home Health Care Serv Q. 2009;28(23):84-99.
37 Jack BW, Chetty VK, Anthony D, Greenwald JL, Sanchez GM, Johnson AE, et al. A reengineered hospital discharge program to decrease rehospitalization: a randomized trial. Ann Intern Med. 2009 Feb;150(3):178-87.

38 Thomas-Hawkins C, Flynn L, Clarke SP. Relationships between registered nurse staffing, processes of nursing care, and nurse-reported patient outcomes in chronic hemodialysis units. Nephrol Nurs J. 2008 Mar-Apr;35(2): 123-30.

39 Foley RN, Hakim RM. Why is the mortality of dialysis patients in the United States much higher than the rest of the world? J Am Soc Nephrol. 2009 Jul;20(7):1432-5.

40 Stone PW, Pogorzelska M, Kunches L, Hirschhorn LR. Hospital staffing and health care-associated infections: a systematic review of the literature. Clin Infect Dis. 2008 Oct;47(7):937-44.

41 Aiken LH, Clarke SP, Sloane DM, Sochalski J, Silber JH. Hospital nurse staffing and patient mortality, nurse burnout, and job dissatisfaction. JAMA. 2002 Oct;288(16):1987-93.

42 Stratton KM, Blegen MA, Pepper G, Vaughn T. Reporting of medication errors by pediatric nurses. J Pediatr Nurs. 2004 Dec;19(6): 385-92.

43 Dunton N, Gajewski B, Taunton RL, Moore J. Nurse staffing and patient falls on acute care hospital units. Nurs Outlook. 2004 Jan-Feb; 52(1):53-9.
44 Thomas-Hawkins C, Denno M, Currier H, Wick G. Staff nurses' perceptions of the work environment in freestanding hemodialysis facilities. Nephrol Nurs J. 2003 Apr;30(2):169_ 78.

45 Patrician PA, Pryor E, Fridman M, Loan L. Needlestick injuries among nursing staff: association with shift-level staffing. Am J Infect Control. 2011 Aug;39(6):477-82.

46 Wolfe WA. Adequacy of dialysis clinic staffing and quality of care: a review of evidence and areas of needed research. Am J Kidney Dis. 2011 Aug;58(2):166-76.

47 Wolfe WA. Is it possible to reduce hospitalizations through evidence-based clinic staffing? Nephrol News Issues. 2016;Epub July 6 [cited 2018 Aug]. Available from: https:// www.healio.com/nephrology/practice-management/news/online/\%7b0dd2aa31-85284f99-b266-34c0f30df3df\%7d/is-it-possibleto-reduce-hospital-admissions-through-evidence-based-clinic-staffing

48 Neumann ME. Dialysis staff ratio bill advances in California. Nephrol News Issues. 2017;Epub March 30 [cited 2018 Mar 18]. Available from: https://www.nephrologynews.com/staff-ratio-bill-advances-in-california/

49 Luna T. Union-backed dialysis clinic bill shelved by California lawmaker. Sacramento Bee. 2017;Epub Sept 8 [cited 2018 Mar 18]. Available from: http://www.sacbee.com/ news/politics-government/capitol-alert/article172059677.html

50 Estes JP, Nguyen DV, Chen Y, Dalrymple LS, Rhee CM, Kalantar-Zadeh K, et al. Time-dynamic profiling with application to hospital readmission among patients on dialysis. Biometrics. 2018;(Jun):5. 